\title{
ENHANCING THE HANDLING OF STANDARD SUBSTITUTION WEIGHTS ON A HYDROSTATIC WEIGHING APPARATUS
}

\author{
D. Simões ${ }^{1}$, A. Furtado ${ }^{2}$, J. Pereira ${ }^{2}$, Rui F. Martins ${ }^{3}$ \\ ${ }^{1}$ Faculdade de Ciências e Tecnologia, Universidade Nova de Lisboa, Caparica, Portugal, dm.simoes@campus.fct.unl.pt \\ ${ }^{2}$ Instituto Português da Qualidade (IPQ), I.P., Caparica, Portugal, afurtado@ipq.pt \\ ${ }^{3}$ UNIDEMI, Faculdade de Ciências e Tecnologia, Universidade Nova de Lisboa, Caparica, Portugal, rfspm@fct.unl.pt
}

\begin{abstract}
:
The hydrostatic weighing apparatus is a firstlevel method used to measure the density of solids and liquids. At the Portuguese Institute for Quality the hydrostatic tests for the determination of the liquids' density implies the multiple manual placement of a set of standard substitution weights on apparatus' balance pan, leading to undesirable perturbations in the weighing environment. Also, by this the eccentricity effects on the balance, contribute for an unwanted increase of the measurement uncertainty. To overcome these phenomena, an automated mechanism was designed and implemented, which allows the placing and recollection of the set of standard weights on the balance. To validate the new mechanism, tests to ultrapure water at $20{ }^{\circ} \mathrm{C}$ were executed before and after its implementation and the obtained results were compared.

Despite a 3-fold increase in density measurement uncertainty, mainly due to the exposure of the standard substitution weights to air convection currents, the execution of hydrostatic tests was improved, not only for the fact that the measurements are now carried out quicker, but also for the fact that the operator only needs to intervene once during the whole test. Moreover, the smaller absolute deviation from the reference density value for ultrapure water at $20{ }^{\circ} \mathrm{C}$ results also in a normalised error $E_{\mathrm{n}}$ lower than 1 (0.3), thus validating the designed automated mechanism for the handling of standard substitution weights.
\end{abstract}

Keywords: hydrostatic weighing; density of liquids; automated mechanism; substitution weights

\section{INTRODUCTION}

The hydrostatic weighing is the first-level measurement method used to determine the density of liquids. It is essentially based in the Archimedes' principle, which states that a body, partially or fully immersed in a fluid, is subject to an upward force named buoyancy, equal to the weight of the fluid displaced by the body. Therefore, the loss in its apparent mass is the mass of fluid displaced by the body. In this method, the immersed body is commonly known as "sinker". The buoyancy force, $\mathrm{B}$, can be calculated as follows:

$B=\rho_{\mathrm{L}} V_{\mathrm{S}} g$

where $\rho_{\mathrm{L}}$ is the density of the liquid, in $\mathrm{kg} \mathrm{m}^{-3}, V_{\mathrm{S}}$ the volume of the sinker, in $\mathrm{m}^{3}$, and $g$ the gravitational acceleration, in $\mathrm{m} \mathrm{s}^{-2}$. As the buoyancy force acts against the weight of the sinker, the resulting force measured by a balance to which it is connected results in a smaller value of mass when compared to the mass of the sinker when it is weighed in the air. This smaller value of mass is often named as the "apparent mass". If the real mass $m_{\mathrm{S}}$ and volume $V_{\mathrm{S}}$ of the sinker are known, and its "apparent mass" $m_{\mathrm{S}}^{\mathrm{a}}$ determined by hydrostatic weighing, the density of the liquid in which the sinker is immersed can be calculated according to equation (2).

$\rho_{\mathrm{L}}=\frac{m_{\mathrm{S}}-m_{\mathrm{S}}^{\mathrm{a}}}{V_{\mathrm{S}}}$

A measuring instrument or apparatus which uses a comparison method against standards allows a decrease in the uncertainty by eliminating environmental and instrumental variations. In the case of the hydrostatic weighing apparatus, the apparent mass of the sinker is compared against the mass of standard substitution weights. This procedure is named "substitution method". Therefore, the apparent mass of the sinker can be calculated according to equation 5 [1].

$m_{\mathrm{S}}^{\mathrm{a}}=W_{\mathrm{W}}+\left(W_{\text {Sread }}-W_{\text {Wread }}\right)\left(1-\frac{\rho_{\mathrm{A}}}{8000}\right)$

In the previous equation, $W_{\mathrm{W}}$ denotes the mass value of the used set of standard substitution weights, in $\mathrm{kg} ; W_{\text {Sread }}$ and $W_{\text {Wread }}$ denote the readings of the balance when loaded with the sinker and with the set of standard weights, in $\mathrm{kg}$, respectively. The term $\left(1-\frac{\rho_{\mathrm{A}}}{8000}\right)$ represents the air 
buoyancy correction, where $\rho_{\mathrm{A}}$ is the density of air, in $\mathrm{kg} \mathrm{m}^{-3}$, in the weighing region.

The mass value of the used set of standard weights can be calculated based on the parameters given by the respective calibration certificate and according to equation 4 [1].

$W_{\mathrm{W}}=m_{\mathrm{W}}\left(1-\frac{\rho_{\mathrm{A}}}{\rho_{\mathrm{W}}}\right)$

where $m_{\mathrm{W}}$ and $\rho_{\mathrm{W}}$ correspond to the mass and the density values of the standard weights given by their calibration certificate, in $\mathrm{kg}$ and $\mathrm{kg} \mathrm{m}^{-3}$, respectively.

The density of air is calculated with the CIPM2007 formula [2].

\section{MATERIALS AND METHODS}

A hydrostatic weighing apparatus features essentially a balance, suitable for underfloor weighing, an underfloor mounted suspension, with a holder for the sinker, and a vessel which contains the test liquid (Figure 1).

A mechanism for IPQ's hydrostatic weighing apparatus was designed to allow the automated loading/unloading of the standard substitution weights on the balance (Figure 2). This mechanism mainly consists of a fork system, vertically actuated by an electric linear axis, which picks and places a standard disc-shaped weight set on a holder, connected to the hook located underneath the balance. The standard weights are loaded on the fork by a qualified operator. It is needed only once before the density measurement.

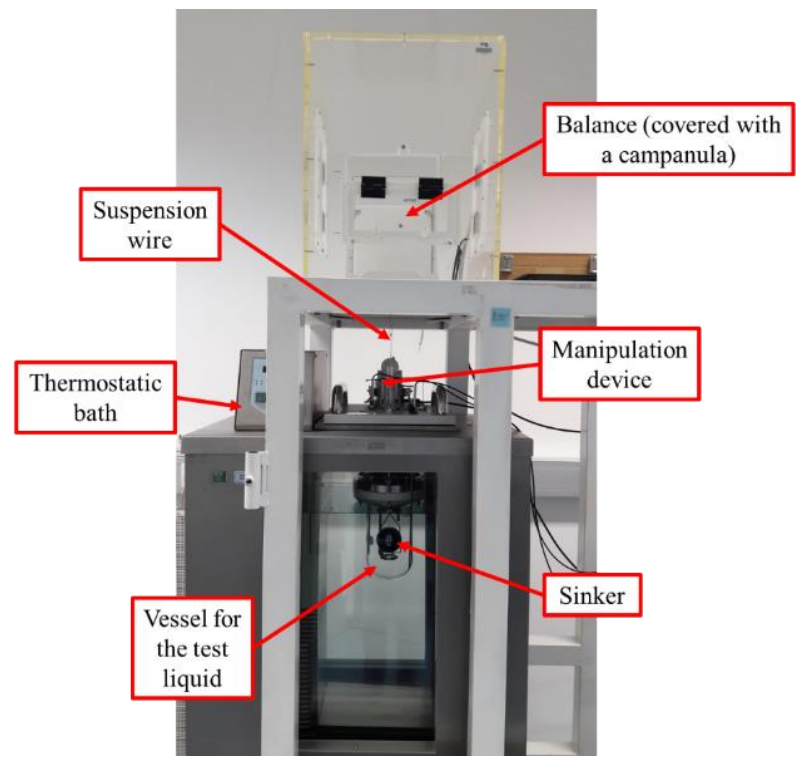

Figure 1: Complete hydrostatic weighing apparatus in use at IPQ.
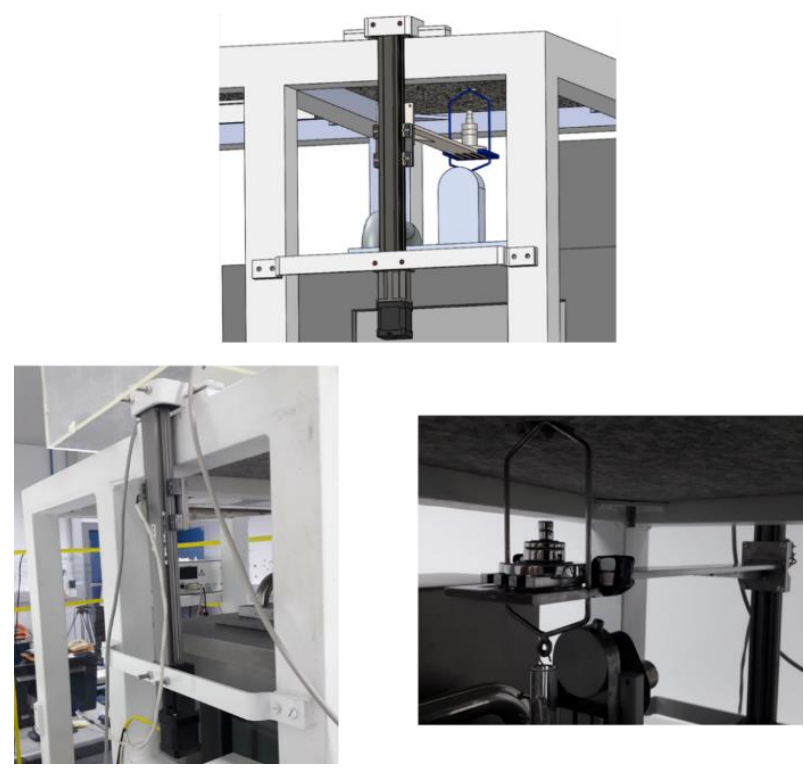

Figure 2: Overview of the automated mechanism designed for the handling of standard substitution weights implemented in IPQ's hydrostatic weighing apparatus.

The mechanism is located between the balance and the thermostatic bath. All the designed components of the mechanism were manufactured in AISI 316 austenitic stainless steel.

The design and simulation of the mechanism were accomplished through the solid modelling computer-aided design (CAD) and computer-aided engineering (CAE) program SolidWorks ${ }^{\circledR}$ and SolidWorks Simulation ${ }^{\circledR}$, respectively.

\subsection{Holder for the Standard Substitution Weights}

The holder for the standard substitution weights (Figure 3) consists in a base, made of a $5 \mathrm{~mm}$ thick rectangular sheet, where the set of standard weights is placed. There are four $8.5 \mathrm{~mm}$ wide slots in the base which allow the passing of the tines of the fork when this element is picking or placing the weights on the holder. The holder sustains the weights through three $2.5 \mathrm{~mm}$ wide tines. In order to accommodate the standard weights, there are two $1.25 \mathrm{~mm}$ deep cavities at the base for the larger diameter discs $(\varnothing 48 \mathrm{~mm}$ and $\emptyset 22 \mathrm{~mm})$, with a $1.5 \mathrm{~mm}$ radial clearance between each cavity and disc.

The holder has a wire structure welded to the base with a circular $\emptyset 2.5 \mathrm{~mm}$ diameter cross-section, having a hook at the top to connect this component to the balance and a ring at the bottom to link it to the suspension. 

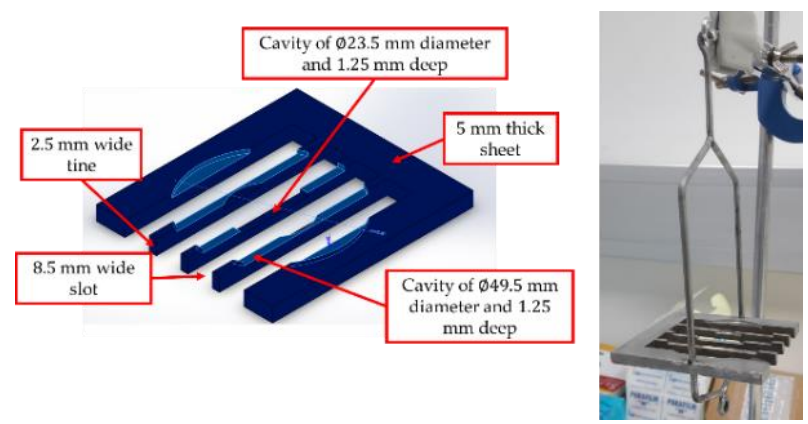

Figure 3: CAD model of the base of the holder for the standard substitution weights (at left) and end-product of the component (at right).

To simulate the stresses and strains on the holder, a static load of $2.5 \mathrm{~N}$ was applied at the surface of the cavity which supports the larger diameter discs. This value corresponds to the mass of the silicon sphere when weighed in the air (approximately $234 \mathrm{~g}$ ) with a safety factor applied. The effect of the gravitational acceleration was also taken into account $\left(g=9.81 \mathrm{~m} \cdot \mathrm{s}^{-2}\right)$.

Figure 4 shows the static stress-strain analysis of the holder for the standard substitution weights.

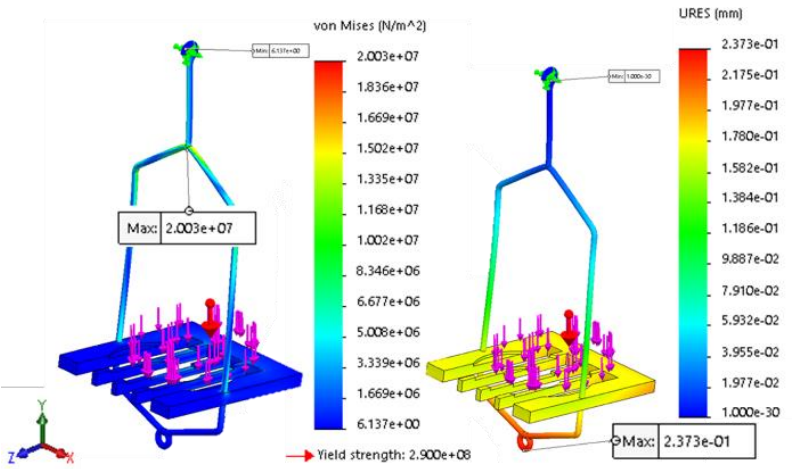

Figure 4: Stress (at left) and strain (at right) analyses of the holder for the standard substitution weights. The deformed shape is not at the real scale.

To prevent the material from yield, the condition given by equation (5) must be verified:

$\sigma_{\mathrm{v}} \leq \sigma_{\max }=\frac{\sigma_{\mathrm{y}}}{S F}$

where $\sigma_{\mathrm{v}}$ is the maximum von Mises stress, in MPa, determined by the Finite Element Method (FEM), $\sigma_{\max }$ is the maximum allowable stress of design, in $\mathrm{MPa}, S F$ is a safety factor of $S F=1.5$ and $\sigma_{\mathrm{y}}$ is the Yield Stress (290 MPa). Given that the maximum value of the von Mises stress, as observed in Figure 4 , is approximately $\sigma_{\mathrm{v}}=20 \mathrm{MPa}$, the von Mises stress is substantially lower than the maximum allowable design stress $\left(\sigma_{\mathrm{v}}=20 \mathrm{MPa} \ll \sigma_{\max }=\right.$ 193.33 MPa), hence concluding that the holder operates in the elastic domain.

Given the required precision to the motion of the fork to allow its tines to pass through the slots of the holder in order to successfully exchange the standard weights between these two components, it is crucial that the displacements caused by the weight of the discs when supported on these elements are not excessive. This factor could potentially lead to an undesirable contact between the fork and the holder, preventing the correct operation of the mechanism. It was verified that the maximum displacement on the holder had a value of approximately $0.24 \mathrm{~mm}$ and is located on the bottom ring for suspension connection. This value does not compromise the functioning of the mechanism.

\subsection{Fork for the Handling of Standard Substitution Weights}

The fork for the handling of standard substitution weights (Figure 5) consists of an arm, manufactured from a $5 \mathrm{~mm}$ thick rectangular sheet with $374.65 \mathrm{~mm}$ long. The end of the arm, where the standard weights are supported, has four tines with a width of $5.5 \mathrm{~mm}$ each, ensuring a total horizontal clearance of $3 \mathrm{~mm}$ between these tines and the existing slots in the holder for the standard weights. This same clearance is also verified between the tines of the holder and the slots in the fork. In order to compensate for any possible misalignment between the fork and the holder from their working positions due to the movement of the mechanism, the existence of these considerable clearances is mandatory. The fork is also provided with two cavities of similar dimensions to the ones designed for the holder of the standard weights.

The fork is connected to the carriage of the electric linear axis through a rectangular sheet of dimensions $60 \mathrm{~mm} \times 54 \mathrm{~mm} \times 5 \mathrm{~mm}$, welded to the arm. This fixing sheet is then fastened to the carriage of the linear axis through four M4 bolts. To reduce the deformations at the end of the fork due to the weight of the discs, a rib was welded at the bottom of this component to the arm and the fixing sheet.
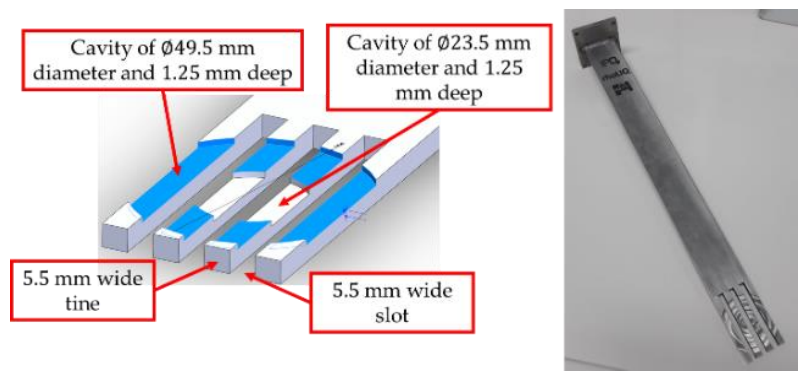

Figure 5: CAD model of the tip of the fork for the handling of standard substitution weights (at left) and the respective end-product (at right).

Figure 6 shows the stress-strain analysis of the entire mechanism (set consisted by the fork, a simplified geometry model of the electric linear axis and its respective fixing bars for fastening the mechanism to the supporting structure of the balance). 


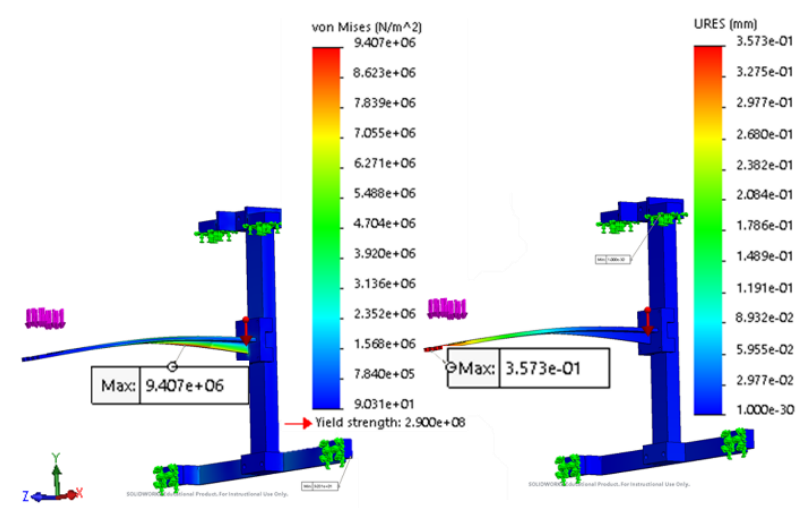

Figure 6: Stress (at left) and strain (at right) analyses of the entire mechanism, consisted by the fork, a simplified geometry model of the electric linear axis and its respective fixing bars. The deformed shape is not at the real scale.

Applying the previous methodology, the von Mises stress is maximum on the rib of the fork with a value of approximately 9.4 MPa. According to the design criteria defined by equation (5), it is concluded that the fork is distant from yielding $\left(\sigma_{\mathrm{v}}=9.4 \mathrm{MPa} \ll \sigma_{\max }=193.33 \mathrm{MPa}\right)$.

The maximum of displacements is on the edge of the fork's tines with a value of $0.36 \mathrm{~mm}$. This is small enough to meet the required precision of the mechanism.

\subsection{Electric Linear Axis}

Based on the reaction forces at the support of the fork, i.e., the fixing sheet, an electric linear axis from Igus ${ }^{\circledR}$ (Figure 7) was chosen for the mechanism for the handling of the standard weights. The linear axis' carriage is driven by a lead-screw, coupled to a NEMA17 Litze stepper motor, being the preferred option for the required precision.

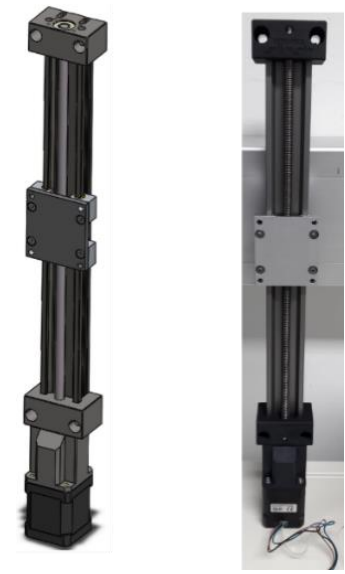

Figure 7: CAD model of the lead-screw driven electric linear axis from Igus ${ }$ (size 0630) (at left); Real model of the electric linear axis (at right).

The NEMA17 Litze stepper motor of the linear axis is controlled through the TB6600 driver, connected to an Arduino UNO R3 board (Figure 8). The Arduino platform was chosen to program the stepper motor because it is an open-source software, having a wide integration with different programming languages, particularly with LabVIEW®. The stepper motor is powered by a $24 \mathrm{~V}$ DC power supply, shown as well in Figure 8.
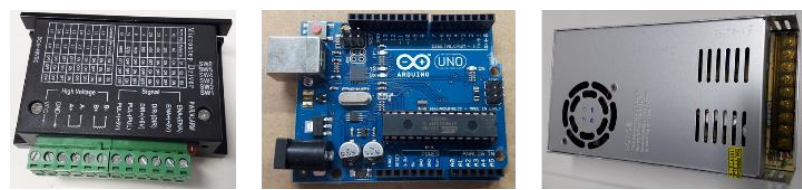

Figure 8: TB6600 stepper motor driver (at left), Arduino UNO R3 board (at the centre) and 24 V DC power supply (at right).

To hold the electronic components in place, and protect the respective connections, these were installed in the interior of a proper aluminium housing (Figure 9).

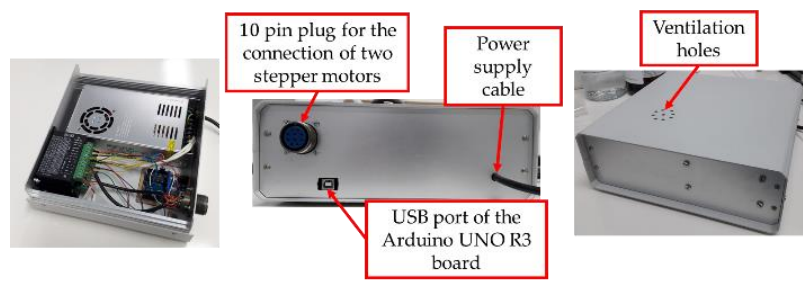

Figure 9: Electric control circuit of the stepper motor mounted in the interior of the housing (at left), existing connections at the back of the housing (at the centre) and ventilation holes on the top of the housing (at right).

The NEMA17 stepper motor of the electric linear axis was programmed using the LINX interface for LabVIEW®. This interface is responsible to establish the connection between the Arduino UNO R3 board and the LabVIEW® software. The developed LabVIEW® program to control the stepper motor was integrated within an already existing program which controls the hydrostatic test, acquiring all the data from the measuring instruments.

It is important to point out that the carriage of the electric linear axis always moves at a constant speed of $0.223 \mathrm{~mm} \cdot \mathrm{s}^{-1}$. It is essential that the actions of placement and removal the standard weight set by the fork are carried out at low speeds to minimise the oscillations in the holder and to prevent the weights from being dropped.

\section{RESULTS AND ITS DISCUSSION}

The validation of the mechanism for the handling of standard weights was performed through the carrying out of hydrostatic tests to ultrapure water (Type 1), at $20{ }^{\circ} \mathrm{C}$. The choice of this liquid was because ultrapure water is a density standard whose properties are well known at a certain temperature range [3]. The sample used in the tests was degassed using a vacuum oven.

The results of density and its respective expanded uncertainty, obtained from the measurements to ultrapure water at $20{ }^{\circ} \mathrm{C}$, both with the hydrostatic weighing apparatus pre and postoptimised, are given in Table 1. The term "pre- 
optimisation" refers to the original apparatus, as it was, while the "post-optimisation" term refers to the improved apparatus with the enhancement of the handling of standard substitution weights. The reference density value for ultrapure water at this temperature, as stated by Tanaka [3], is also listed in the same table.

Table 1: Results of density values $\rho_{L}$ and respective expanded uncertainty $U_{\rho_{L}}$ (absolute and relative values) obtained from measurements with the hydrostatic weighing apparatus, pre and post-optimised, of an ultrapure water sample at $20^{\circ} \mathrm{C}$. The reference value observed by Tanaka [3] is also shown.

\begin{tabular}{|r|c|cc|}
\hline $\begin{array}{r}\text { Ultrapure } \\
\text { Water }\end{array}$ & $\begin{array}{c}\boldsymbol{\rho}_{\mathbf{L}} \\
\left./ \mathbf{~ k g} \cdot \mathbf{m}^{-3}\right)\end{array}$ & $\begin{array}{c}\boldsymbol{U}_{\boldsymbol{\rho}_{\mathbf{L}}} \\
\mathbf{k k g}^{-3} \mathbf{m}^{-3}\end{array}$ & $/ \mathbf{p p m}$ \\
\hline $\begin{array}{r}\text { Pre- } \\
\text { optimisation }\end{array}$ & 998.2193 & $\begin{array}{c}0.0042 \\
(k=1.97)\end{array}$ & 4.2 \\
\hline $\begin{array}{r}\text { Post- } \\
\text { optimisation }\end{array}$ & 998.211 & $\begin{array}{c}0.013 \\
(k=2.07)\end{array}$ & 13.2 \\
\hline Tanaka & 998.2067 & $\begin{array}{c}0.00083 \\
(k=2.00)\end{array}$ & 0.8 \\
\hline
\end{tabular}

Figure 10 illustrates the previous results in a scatter plot to provide for a better comparison between them.

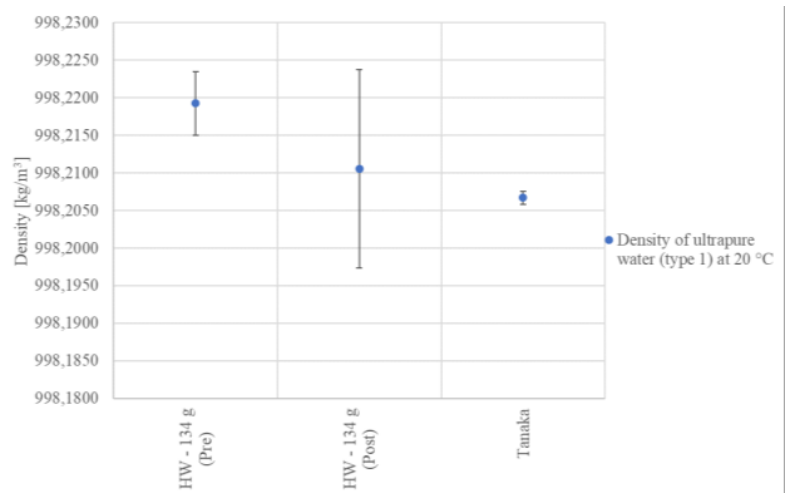

Figure 10: Scatter plot of the density of ultrapure water (Type 1), at $20^{\circ} \mathrm{C}$, measured by the hydrostatic weighing (HW) apparatus pre and post-optimised and the respective value observed by Tanaka [3]. The vertical bars represent the values of expanded uncertainty relative to each measured density value.

Another indicator which allows for a clearer comparison of results is the relative deviation in density $\delta_{\rho}$, in ppm. Considering the density value of ultrapure water, at $20{ }^{\circ} \mathrm{C}$, observed by Tanaka [3] as reference, the relative deviation of the obtained results by hydrostatic weighing from the reference value is given as follows:

$$
\delta_{\rho}=\left|\frac{\rho_{\mathrm{HW}}-\rho_{\text {ref. }}}{\rho_{\text {ref. }}}\right| \times 10^{6}
$$

where $\rho_{\mathrm{HW}}$ and $\rho_{\text {ref. }}$ are the density values of the liquid experimentally measured by hydrostatic weighing and determined by Tanaka, respectively. The term $\left|\rho_{\mathrm{HW}}-\rho_{\text {ref. }}\right|$ can be described as the absolute deviation in density, $\Delta \rho$.
The validation procedure of a certain measuring method implies the calculation of the normalised error $E_{\mathrm{n}}$ of a measurement result against a reference value. The results are satisfactory if the normalised error is less than or equal to 1 , i.e., $E_{\mathrm{n}} \leq 1$. Assuming once again the density value for ultrapure water at $20^{\circ} \mathrm{C}$ determined by Tanaka [3] as reference, the normalised error yielded by the results obtained by hydrostatic weighing can be calculated according to equation (7).

$$
E_{\mathrm{n}}=\frac{\left|\rho_{\mathrm{HW}}-\rho_{\text {ref. }}\right|}{\sqrt{U_{\rho_{\mathrm{HW}}}{ }^{2}+U_{\rho_{\text {ref. }}{ }^{2}}^{2}}}
$$

In the equation above, $U_{\rho_{\mathrm{HW}}}$ and $U_{\rho_{\text {ref. }}}$ are the expanded uncertainty values in the measurements by hydrostatic weighing and in the reference value, observed by Tanaka [3], respectively.

The absolute and relative deviations in density, of the obtained results by hydrostatic weighing to ultrapure water at $20^{\circ} \mathrm{C}$ from the reference value observed by Tanaka [3], both in the stages of pre and post-optimisation of the apparatus, are listed in Table 2. The normalised error of the method is also shown.

Table 2: Absolute, $\Delta \rho$, and relative, $\delta_{\rho}$, deviations in density, of the obtained results by hydrostatic weighing to ultrapure water (type 1 ) at $20^{\circ} \mathrm{C}$ from the reference value observed by Tanaka [3], both in the stages of pre and post-optimisation of the apparatus. The normalised error, $E_{n}$, is also listed.

\begin{tabular}{|r|c|c|c|}
\hline Stage & $\begin{array}{c}\Delta \boldsymbol{\rho} \\
/ \mathbf{~ k} \cdot \mathbf{m}^{-3}\end{array}$ & $\begin{array}{c}\boldsymbol{\delta}_{\boldsymbol{\rho}} \\
/ \mathbf{p p m}\end{array}$ & $\boldsymbol{E}_{\mathbf{n}}$ \\
\hline $\begin{array}{r}\text { Pre- } \\
\text { optimisation }\end{array}$ & 0.0126 & 12.6 & 2.9 \\
\hline $\begin{array}{r}\text { Post- } \\
\text { optimisation }\end{array}$ & 0.004 & 3.9 & 0.3 \\
\hline
\end{tabular}

Comparing the results obtained in the two different stages, with the reference value suggested by Tanaka [3], a smaller absolute deviation in the density of the liquid $\Delta \rho$, was verified in the stage of post-optimisation $\quad\left(0.004 \mathrm{~kg} \mathrm{~m}^{-3} \quad(3.9 \mathrm{ppm})\right)$ relatively to the pre-optimisation $\left(0.0126 \mathrm{~kg} \mathrm{~m}^{-3}\right.$ $(12.6 \mathrm{ppm}))$. These results yield in a normalised error $E_{\mathrm{n}}$ of 2.9 for the stage of pre-optimisation of the apparatus and 0.3 for the stage of postoptimisation. The main factor which could have potentially led to this difference is the fact that the ultrapure water sample used, despite being the same at pre and post- optimisation tests, may varied in its gas saturation [3], due to different ambient conditions, and therefore resulting in different density deviations from the Tanaka's reference value. There are other possible causes which could lead to a more significant deviation in density from the reference value, such as the lack of knowledge of the chemical composition of the sample (in 
particular, the isotopic composition) [3]; or other unknown factors, such as mathematical distributions, heat or contamination of the sample.

Concerning the density expanded uncertainty of the liquid $U_{\rho_{\mathrm{L}}}$, a value approximately 3 times greater was verified in the hydrostatic tests after the optimisation of the apparatus $\left(0.013 \mathrm{~kg} \mathrm{~m}^{-3}\right)$ when compared against the value obtained from the preoptimisation stage $\left(0.0042 \mathrm{~kg} \mathrm{~m}^{-3}\right)$. This increase was mainly caused by a greater standard uncertainty in the repeatability of the readings of the balance $u_{\text {rep. }}$, in the stage of post-optimisation $\left(2.6 \times 10^{-4} \mathrm{~g}\right)$ when compared against the pre-optimisation stage $\left(5.0 \times 10^{-5} \mathrm{~g}\right)$. This phenomenon was primarily due to the exposure of the holder and respective standard weights to convection currents and vibrations from the bath, disturbing the stability of the set and leading to unstable readings of the balance.

Another modification made to IPQ's hydrostatic weighing apparatus after its optimisation was the installation of the pressure, temperature and relative humidity sensors for the atmospheric air in the operating area of the mechanism for the handling of standard weights. These sensors were previously installed in the interior of the campanula which covers the balance, so they had to be moved to a location closer to where the standard weights are now placed in order to yield measurements with a higher reliability degree, resulting in a more precise calculation of the buoyancy force originated by the air. Given that the operation region of the mechanism is exposed to the surrounding environment, a significant variation in the air conditions within this region may occur, leading to a greater dispersion in the measurements of this component, thus, resulting in a larger uncertainty in repeatability. Nonetheless, a slightly smaller uncertainty in the measurements of the air was still verified in the stage of post-optimisation of the apparatus.

\section{CONCLUSION}

Despite a 3-fold increase in density measurement uncertainty $U_{\rho_{L}}$, from $0.0042 \mathrm{~kg} \mathrm{~m}^{-3}$ to $0.013 \mathrm{~kg} \mathrm{~m}^{-3}$, the smaller absolute deviation from the reference density value for ultrapure water at $20{ }^{\circ} \mathrm{C}$ results also in a satisfactory normalised error $E_{\mathrm{n}}$ of 0.3 , thus validating the designed automated mechanism for the handling of standard substitution weights. Furthermore, the execution of hydrostatic tests was improved with the introduction of a mechanism for the handling of standard weights, not only by the fact that the measurements are now carried out quicker, but also that the operator only needs to intervene once during the whole test. A better stabilisation of the weighing environment (i.e., in air conditions) was also observed, leading to a slight decrease in the uncertainty in the density of the liquid.

\section{ACKNOWLEDGEMENT}

This work was developed under the framework of the EMPIR project "17RPT02-rhoLiq" which is carried out with funding of European Union under the EMPIR. The EMPIR is jointly funded by the EMPIR participating countries within EURAMET and the European Union.

\section{REFERENCES}

[1] H. Fehlauer, H. Wolf, "Density reference liquids certified by the Physikalisch-Technische Bundesanstalt", Meas. Sci. Technol., vol. 17, pp. 2588-2592, 2006.

[2] A. Picard, R. S. Davis, M. Gläser, K. Fujii, "Revised formula for the density of moist air (CIPM-2007)”, Metrologia, vol. 45, pp. 149-155, 2008.

[3] M. Tanaka, G. Girard, R. Davis, A. Peuto, N. Bignell, "Recommended table for the density of water between $0{ }^{\circ} \mathrm{C}$ and $40^{\circ} \mathrm{C}$ based on recent experimental reports", Metrologia, vol. 38, pp. 301-309, 2001. 